\title{
Programming Light-Harvesting Efficiency Using DNA Origami
}

\author{
Elisa A. Hemmig, ${ }^{\dagger}$ Celestino Creatore, ${ }^{\dagger}$ Bettina Wünsch, ${ }_{\dagger}^{\ddagger}$ Lisa Hecker, ${ }^{\ddagger}$ Philip Mair, ${ }^{\dagger}$ M. Andy Parker, ${ }^{\dagger}$
} Stephen Emmott, ${ }^{\text {II }}$ Philip Tinnefeld, ${ }^{\dagger}$ Ulrich F. Keyser, ${ }^{* \dagger}{ }^{\dagger}$ and Alex W. Chin ${ }^{* \dagger}$

${ }^{\dagger}$ Cavendish Laboratory, University of Cambridge, Cambridge CB3 0HE, United Kingdom

${ }^{\ddagger}$ Institut für Physikalische und Theoretische Chemie, TU Braunschweig, 38106 Braunschweig, Germany

${ }^{\text {II }}$ Computational Science Laboratory, Microsoft Research, Cambridge CB1 2FB, United Kingdom

\section{Supporting Information}

ABSTRACT: The remarkable performance and quantum efficiency of biological light-harvesting complexes has prompted a multidisciplinary interest in engineering biologically inspired antenna systems as a possible route to novel solar cell technologies. Key to the effectiveness of biological "nanomachines" in light capture and energy transport is their highly ordered nanoscale architecture of photoactive molecules. Recently, DNA origami has emerged as a powerful tool for organizing multiple chromophores with base-pair accuracy and full geometric freedom. Here, we present a programmable antenna array on a DNA origami platform that enables the implementation of rationally designed antenna structures. We systematically analyze the light-harvesting efficiency with respect to number of donors and interdye distances of a ring-like antenna using ensemble and single-molecule fluorescence spectroscopy and detailed Förster modeling. This comprehensive study demonstrates exquisite and reliable structural control over multichromophoric geometries and points to DNA origami as highly versatile platform for testing design concepts in artificial light-harvesting networks.

KEYWORDS: DNA nanotechnology, DNA origami, artificial light-harvesting, Förster resonance energy transfer, fluorescence spectroscopy
$\mathrm{B}$ iological light-harvesting complexes found in photosynthetic organisms efficiently capture solar photons by exploiting networks of precisely positioned chromophores. Such chromophore assemblies feed multiple electronic excitations into a single reactive center via nonradiative Förster resonance energy transfer (FRET), leading to substantial absorption amplification. Energy transfer is further promoted by delocalized exciton states and quantum coherent transport. This allows the subsequent biochemical reactions, which are driven by electron-transfer events, to thrive even at ultralow light conditions. ${ }^{1,2}$ Although the underlying design principles of such natural photocells have been studied for over a century, ${ }^{1}$ engineering synthetic analogues remains a formidable challenge.

The ability to use DNA as a template to arrange functional elements such as organic dyes ${ }^{3-5}$ into specific arrays has undergone remarkable development over the last few decades. Recently, such chromophore-labeled DNA structures have been successfully employed to create photonic wires ${ }^{5-7}$ and antenna systems, ${ }^{8-11}$ marking the advent of DNA nanotechnology in the field of artificial photosynthesis. The programmed selfassembly of DNA has also inspired the development of structure-based computational models for light-harvesting applications. ${ }^{12}$ Most of the studies so far, however, were performed using small DNA tile structures ${ }^{8}$ or DNA constructs with flexible arms ${ }^{7}$ - which, though establishing the crucial proofs of concept, are likely to suffer from low stability, sample heterogeneities, and structural uncertainties. With such high levels of variability in these structures, characterizing their properties requires sophisticated single-molecule detection; successful engineering of a desired function cannot be easily inferred from ensemble measurements. Moreover, in many of these DNA-chromophore structures, ${ }^{7}$ the light-harvesting topology is dictated by the underlying DNA template. This work demonstrates how many of these constraints can be removed by using programmable DNA origami structures to spatially coordinate dyes and effectively implement theoretical models of antenna systems in a highly robust and reproducible way. ${ }^{12}$

The recent development of DNA origami based selfassembly ${ }^{13,14}$ has enabled the fabrication of DNA nanostructures with significantly greater mechanical stability and rigidity. ${ }^{15}$ DNA origami structures are formed by folding a long, single-stranded piece of DNA ("scaffold") into a designed shape with the aid of hundreds of short, complementary "staple" strands. Each staple is chemically addressable for

Received: December 16, 2015

Revised: February 22, 2016

Published: February 23, 2016 


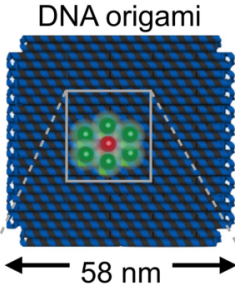

Donor (Cy3)

Acceptor (Cy5)

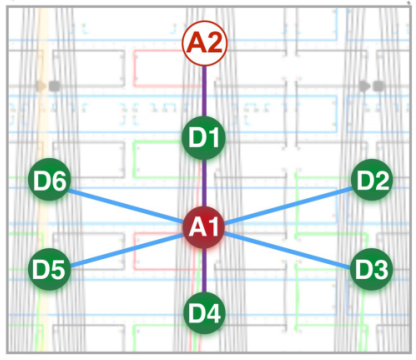

Estimated next-neighbour distance $-5.2 \mathrm{~nm}$

$-5.6 \mathrm{~nm}$

b

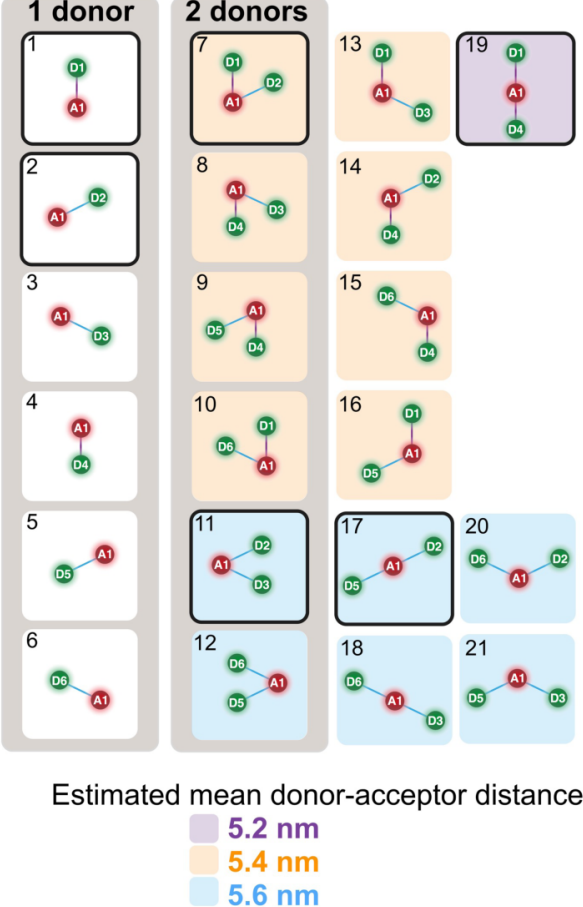

3 donors

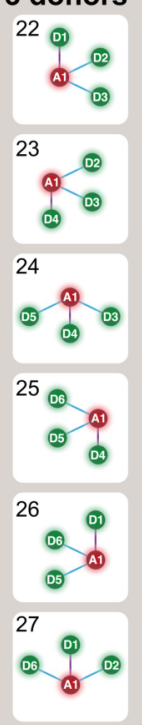

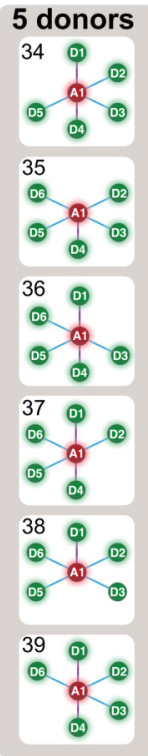

0 donors

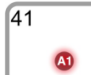

6 donors

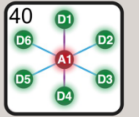

Adjacent donor geometries

Control sample

Single-molecule measurements

Figure 1. Systematic design of DNA origami based donor-acceptor geometries. (a) Ring of Cy3 donor dyes (green) surrounds a Cy5 acceptor dye (red) on a flat, square-shaped DNA origami platform. The zoomed-in window shows the precise fluorophore attachment sites using a caDNAno scheme. (b) For each number of donors $N$, we created all six permutations of adjacent donor positions (gray boxes) and prepared a control sample containing an acceptor only (gray frame). For $N=2$, we assembled all the possible dye geometries resulting in three groups of different mean donor-acceptor distances (colored boxes). The seven structures highlighted with black frames are analyzed both in ensemble and single-molecule measurements.

functionalization with fluorescent tags. To demonstrate how antenna designs can be implemented and analyzed, we consider the problem of a single acceptor dye to which we progressively couple a number of donor dyes. For a set minimum donoracceptor separation, we would expect that the total donoracceptor energy transfer under FRET would be maximized by introducing each extra donor at this minimum separation; in $2 \mathrm{D}$, the optimal configuration would be a ring. Indeed, a circular ring of donors enclosing an acceptor (reaction center) is a motif found in the (2D) photosynthetic membranes of purple bacteria. ${ }^{16}$ To create this antenna geometry, we designed a two-layered DNA origami platform (170 bp $\times 24$ helices), and selected eight fluorophore attachment sites on this "breadboard" so that the fluorophore is oriented away from the plane of the DNA platform. Six of these sites (Cy3 attachment) form a donor ring that surrounds a central acceptor site ( $\mathrm{Cy} 5$ attachment). A further $\mathrm{Cy} 5$ attachment site is positioned outside the ring but equidistant from the nearest donor as the central acceptor. The well-defined DNA lattice allows us to estimate interchromophore distances using singlemolecule measurements of the energy transfer efficiency of single donor-acceptor pairs. This yields distances of $\sim 5.2 \mathrm{~nm}$ in a perpendicular and $\sim 5.6 \mathrm{~nm}$ in a diagonal orientation with respect to helix direction (Figure $1 \mathrm{a}$ and Supporting Information (SI) Note S3). For this particular FRET pair (Cy3-Cy5) the Förster radius is $R_{0}=5.4 \mathrm{~nm}:{ }^{17}$ the length scales of our platform are thus consistent with having FRET as the appropriate theory for energy transfer. At such interdye separations, the dyes are weakly coupled, the coupling being weak with respect to the homogeneous spectral line broadening. Hence, the transfer of the excitation energy between the donor and acceptor dyes can be correctly described within the FRET theory. ${ }^{12,18,19}$ The detailed design, the protocols for assembly and purification, as well as the characterization of the DNA origami platforms have been reported earlier. ${ }^{20}$

A key advantage of the DNA origami technique is its modularity; any of the fluorescently labeled staples can be simply replaced with their original, unmodified counterparts. Arbitrary combinations of fluorophores can therefore be programmed on the predefined positions on the DNA origami platform (see SI Note S1 for DNA sequences of the modified staple strands and chemical structure of the dye attachment). Exploring this combinatorial freedom, we assembled over 30 structures by systematically varying the number of (adjacent) donors and permuting their positions (Figure $1 \mathrm{~b}$ ). In the class of two-donor samples, we created all possible dye geometries yielding groups of different mean donor-acceptor distances (Figure 1b). The DNA origami approach thus enables us to overcome the shortcomings of the antenna structures mentioned earlier ${ }^{7}$ and explore the rational design of lightharvesting building blocks.

We begin with assessing the effectiveness of directional funnelling of excitation energy from the ring of donor molecules to the acceptor molecule in the center. The acceptor molecule is positioned inside $\left(\operatorname{ring} A_{1}\right)$ or outside $\left(\operatorname{ring} A_{2}\right)$ the ring (Figure 2a). Upon excitation of the donors, we observe a clearly enhanced fluorescence emission of the acceptor in ring $A_{1}$ as compared to ring $A_{2}$. The fluorescence emission spectra of the control samples $D_{1}-A_{1}$ and $D_{1}-A_{2}$ are nearly identical (Figure $2 \mathrm{~b}$ ), which confirms that the result of Figure 2a is not induced by underlying structural differences of the DNA origami platform at these two acceptor positions. The excitation 

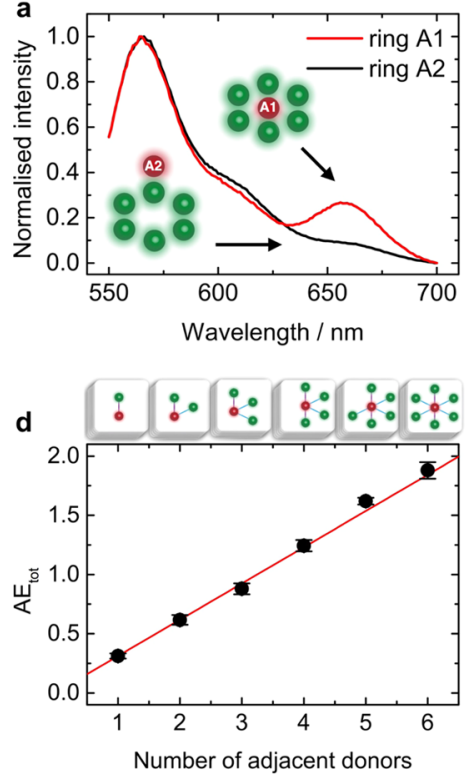
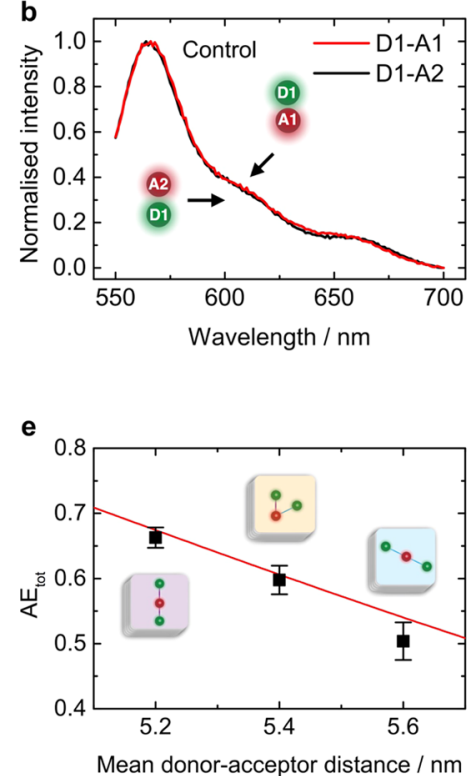

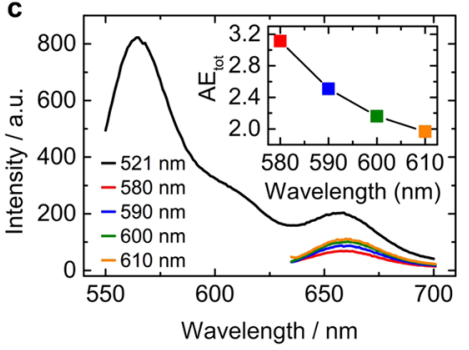

f

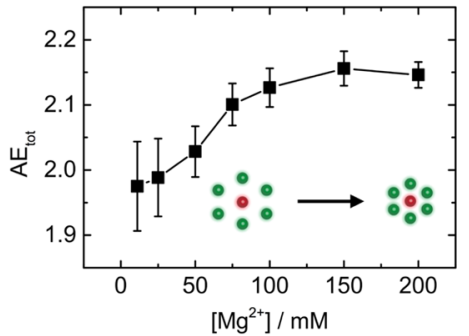

Figure 2. Light-harvesting efficiency depends on donor-acceptor geometry. (a) Normalized fluorescence emission spectra with acceptor inside (ring $A_{1}$, red line) and outside (ring $A_{2}$, black line) the donor ring. (b) Normalized fluorescence emission spectra of corresponding donor-acceptor pairs with donor fixed at $D_{1}$ and acceptor at $A_{1}\left(D_{1}-A_{1}\right.$, red line) and at $A_{2}\left(D_{1}-A_{2}\right.$, black line). (c) Fluorescence emission spectra of the six-donor ring with an acceptor in the center when excited at different wavelengths. The black line corresponds to the emission when excited at the Cy3 (donor) excitation wavelength at $521 \mathrm{~nm}$. The colored lines correspond to the emission at direct Cy5 (acceptor) excitation at different wavelengths in the range $580-610 \mathrm{~nm}$. Inset: Antenna effect $\left(\mathrm{AE}_{\text {tot }}\right)$ as a function of the acceptor excitation wavelength. (d) $\mathrm{AE}_{\text {tot }}$ in dependence of number of adjacent donors $N$ with linear fit (red line) according to eq 4 . For each $N$, we averaged over all possible combinations of adjacent donors in the ring (Figure $1 b)$. The error bars correspond to the standard error of the mean. (e) $\mathrm{AE}_{\text {tot }}$ as a function of mean donor-to-acceptor distance as determined from the two-donor samples (Figure 1b). We prepared three independent replicates of each sample type. The error bars correspond to the standard error of the mean. The function (red line) shows the theoretical distance dependence of $\mathrm{AE}_{\text {tot }}$ (eq 5). (f) Influence of the concentration of $\mathrm{Mg}^{2+}$ ions in the buffer solution on $\mathrm{AE}_{\text {tot }}$ for the six-donor ring with an acceptor in the center. An increase in $\mathrm{Mg}^{2+}$ ion concentration is known to shrink the interhelix distances in DNA origami structures. ${ }^{20}$ We prepared three independent replicates of the sample. The error bars correspond to the standard error of the mean.

concentration observed in ring $A_{1}$ thus demonstrates the viability of our antenna system.

Building on this, we systematically study the light-harvesting efficiency of our DNA-based structures, which is quantified by the antenna effect $\left(\mathrm{AE}_{\text {tot }}\right){ }^{21}$ This is defined as the ratio between the fluorescence of the acceptor upon excitation of donors $\left[I_{\mathrm{A}}\left(D^{*}\right)\right]$ to the fluorescence of the acceptor upon its direct excitation $\left[I_{\mathrm{A}}\left(A^{*}\right)\right]$ at given excitation wavelengths

$$
\mathrm{AE}_{\mathrm{tot}}=\frac{I_{\mathrm{A}}\left(D^{*}\right)}{I_{\mathrm{A}}\left(A^{*}\right)}
$$

The antenna effect quantifies the acceptor emission due to energy transfer from neighboring donors relative to acceptor emission resulting from its direct excitation, assuming equal photon fluxes incident on both donor and acceptor dyes (see SI Figure S4 for more details on the analysis of the fluorescence emission spectra). It is important to highlight that the antenna effect is a parameter that can be tuned with the excitation wavelengths used. As shown in Figure $2 \mathrm{c}, \mathrm{AE}_{\text {tot }}$ decreases as the acceptor excitation wavelength approaches the absorption maximum of Cy5 (SI Figure S2) when the donor excitation wavelength is fixed.

We first analyze the antenna effect at an increasing number of donors $N$ enclosing the common acceptor; for each donor number, $\mathrm{AE}_{\text {tot }}$ is obtained by averaging over all possible arrangements of adjacent donors within the ring (Figure $1 \mathrm{~b}$ ). This approach ensures self-averaging of local donor-DNA and donor-acceptor interactions over the ring. For the fully occupied ring $(N=6)$, three replicates are prepared. Furthermore, $\mathrm{AE}_{\text {tot }}$ is corrected for direct excitation of the acceptor at the donor wavelength $(521 \mathrm{~nm})$ using a sample containing an acceptor only $\left(\mathrm{AE}_{\text {tot }}^{0} \approx 0.08\right)$, see Figure $1 \mathrm{~b}$. We observe a linear increase of $\mathrm{AE}_{\text {tot }}$ with donor number $N$, which exceeds unity for $N>3$ with a maximum value of $\sim 2$ for $N=6$ (Figure 2d). This result implies that a higher total intensity of light is released by the acceptor dye following excitation of the donors $(521 \mathrm{~nm})$ than by direct excitation of the acceptor $(600$ $\mathrm{nm}$ ). This is in good agreement with a theoretical analysis (see Model in Methods and the SI Note S8) based on the solution of the rate equations of the excited state populations of donor and acceptor chromophores interacting via FRET, which predicts a linear scaling of $\mathrm{AE}_{\text {tot }}$ with number of donors $N$; see eq 4. Figure $2 \mathrm{~d}$ shows how the experimental $\mathrm{AE}$ values are linearly fitted by eq 4, whereas in the SI Note S7, we study the dependence of $\mathrm{AE}_{\text {tot }}$ on the number of donors without introducing any fitting parameters.

As expected, energy diffusion between the donors (homoFRET) does not alter the net $\mathrm{AE}_{\text {tot }}$ in our dye configurations (see the Model section in Methods and the SI Note S8). In contrast to a homo-FRET based photonic wire, ${ }^{10}$ the ring geometry ensures that all donors can effectively transport energy to the acceptor. Hence, a circular antenna system consistently outperforms an equivalent linear array with the acceptor at one end, where the antenna effect is highly susceptible to weak links or disorder in the chain of dyes. Furthermore, $\mathrm{AE}_{\text {tot }}$ rapidly saturates as a function of $N$ due to 

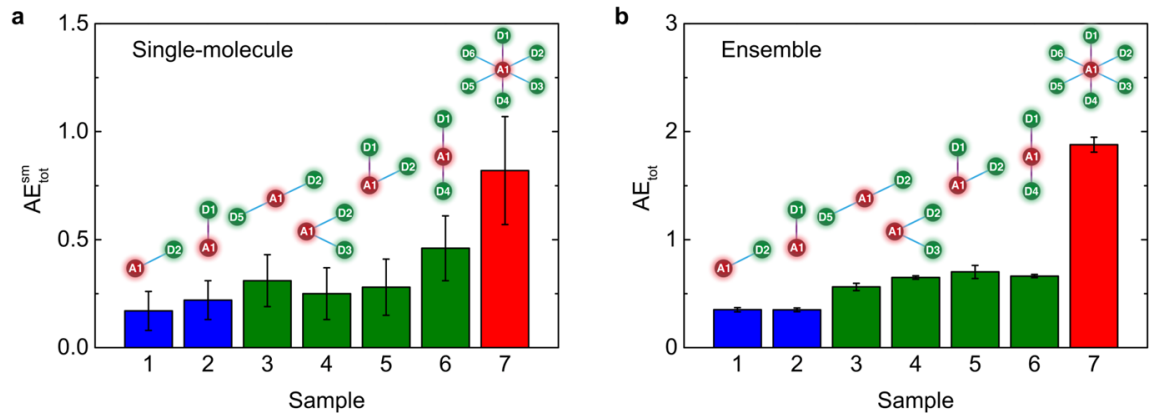

Figure 3. Direct comparison between single-molecule and ensemble fluorescence measurements. (a) Antenna effect ( $\left.\mathrm{AE}_{\text {tot }}^{\mathrm{sm}}\right)$ obtained from singlemolecule measurements. We analyzed one-donor (blue), two-donor (green), and six-donor (red) samples (Figure 1b). We screened several thousand molecules for each sample type, and used a Gaussian fit to determine the antenna effect. The error bars correspond to the standard deviation of the Gaussian fit. (b) Antenna effect $\left(\mathrm{AE}_{\mathrm{tot}}\right)$ obtained from ensemble measurements. We analyzed one-donor (blue), two-donor (green), and six-donor (red) samples (Figure 1b). Each sample was prepared in three independent replicates. The error bars correspond to the standard error of the mean.

the inefficiency of the diffusive homo-FRET required to transfer energy across the array to the acceptor (see SI Note S9).

Having understood the collective role played by the donors, we analyze how small changes in the donor-acceptor separation affect the light-harvesting efficiency of our antenna. We are able to vary the donor-acceptor separation in two fundamentally different ways; first, we can classify all possible two-donor configurations according to their mean donoracceptor distance (Figure 1b) as imposed by the anchoring positions on the DNA origami platform, which yields three different mean donor-acceptor distances (Figure 1b). As shown in Figure $2 \mathrm{e}$, an increase in this distance by $\lesssim 1 \mathrm{~nm}$ results in a substantial decrease of $\mathrm{AE}_{\text {tot }}$ by $\sim 23 \%$. This is in agreement with the theoretical prediction given by eq 5 . Second, we can dynamically tune the intrinsic compactness of a DNA origami structure and thereby the distances between the attached fluorophores by varying the concentration of $\mathrm{Mg}^{2+}$ ions in the buffer solution. ${ }^{20}$ Increasing the concentration of $\mathrm{Mg}^{2+}$ ions leads to reduced interhelix distances due to electrostatic screening of the negatively charged DNA backbone. Indeed, as the $\mathrm{MgCl}_{2}$ concentration is increased from 11 to $200 \mathrm{mM}, \mathrm{AE}_{\text {tot }}$ is enhanced by $\sim 9 \%$ for the full donor-ring antenna (see Figure $2 \mathrm{f}$ and SI Note S5 for more details).

As an essential part of testing new structures, we verify the results obtained in bulk using measurements of the antenna effect at single-molecule level for a subset of samples (Figure 1b). The experimental setup applies alternating laser excitation (ALEX) of donor and acceptor fluorophores on diffusing molecules with separate donor and acceptor detection channels recording fluorescence bursts from single molecules (see Methods). This technique allows sorting of subpopulations and disregard molecules without FRET signal in the analysis. The quantity equivalent to $A E_{\text {tot }}$ is defined as $A E_{\text {tot }}^{\mathrm{sm}}=\mathrm{GR} / \mathrm{RR}$, where GR corresponds to "green" excitation-"red" detection and RR to "red" excitation-"red" detection. We select representative samples with donor numbers $N=1,2,6$ and measure the antenna effect both in single-molecule (Figure 3a) and bulk (Figure 3b) measurements (see SI Note S6 for a comparison of energy transfer efficiency values). The overall trend, increasing antenna effect with donor number, is qualitatively reproduced in the single-molecule measurements. Furthermore, at a given donor number, we observe an increase in antenna effect with decreasing mean donor-acceptor distance (e.g., sample 3 vs sample 6) (Figure 3a). When averaging over all the values within a sample set with equal donor number $(N=1,2,6)$, the discrepancy in the mean values of $\mathrm{AE}_{\text {tot }}$ and $\mathrm{AE}_{\text {tot }}^{\mathrm{sm}}$ can be clearly explained by the different excitation wavelengths used for the acceptor molecule in the respective experiment (see Figure 2c and SI Note S6 for a detailed explanation). Importantly, the single-molecule measurements show remarkably homogeneous populations for each sample indicating that all DNA origami structures have the same number of donors within our measurement accuracy (see the stoichiometry histograms in SI Note S6). This gives us high confidence on the homogeneity and robustness of the DNA origami structures. In addition, the width of the homogeneous populations of the antenna effect (SI Figure S6.1a) is mainly dominated by shot-noise and no significant conformational heterogeneity can be detected. The high quality of samples and close agreement between the results obtained from both techniques thus allow the bulk measurements to be used as effective analytical tool.

To conclude, we have systematically built up a circular antenna system according to a rational design on a programmable material with nanometer precision. Our rigorous and quantitative analysis has verified that the ring geometry functions effectively as an antenna. We have convincingly shown that DNA origami is a highly reliable tool for generating these antennas. Compared to DNA structures with flexible $\mathrm{arms}^{7}$, we have rigidified the system as inspired by protein scaffolds in real biological antennas; this promising avenue could be further explored using higher dimensional DNA origami architectures. Moreover, we have created a versatile platform for unravelling optimal energy transfer networks: by placing more dyes, such as multiple acceptors, we could investigate optimal donor-to-acceptor ratios or network geometries with multiple dye types, and even begin to replicate analogues of the energy transport system of entire photosynthetic membranes.

An intriguing future direction of this work is the possibility of engineering quantum effects that alter the fundamental nature of the antenna's photoexcitations. Recent theoretical work suggests that such effects could be exploited to enhance the performance of biologically inspired light-harvesting systems. ${ }^{2-25}$ Quantum states, such as coherently coupled dimers, have already been realized in simple DNA duplexes, ${ }^{26}$ but control over their spatial organization proves to be challenging. The ability to program multichromophoric geometries using DNA origami could increase the complexity and connectivity of 
such quantum states, potentially creating an antenna operating beyond the classical regime.

Methods. Steady-State Fluorescence Measurements in Bulk. Steady-state fluorescence emission was measured in bulk using a Cary Eclipse Fluorescence Spectrophotometer (Agilent Technologies) and a low volume cuvette $(50 \mu \mathrm{L})$ (SigmaAldrich). Donor molecules (Cy3) were excited at $521 \mathrm{~nm}$. Direct excitation of acceptor molecules (Cy5) was achieved using an excitation wavelength of $600 \mathrm{~nm}$. The excitation and emission slits were set to $20 \mathrm{~nm}$ for the DNA origami samples $\left(\sim 2 \mathrm{nM}\right.$ in $11 \mathrm{mM} \mathrm{MgCl}_{2}$, buffered with $\left.0.5 \times \mathrm{TBE}\right)$ and to 10 $\mathrm{nm}$ for the Cy3- and Cy5-labeled staple strands $(\sim 500 \mathrm{nM}$ in 1 $\times \mathrm{TE})$, respectively. Emission spectra were collected over a wavelength range of 550-700 nm upon excitation at $521 \mathrm{~nm}$, and $635-700 \mathrm{~nm}$ upon excitation at $600 \mathrm{~nm}$, respectively. For each sample, five fluorescence emission spectra were recorded and averaged for further analysis. The magnesium chloride titration was performed by stepwise addition of $1 \mathrm{M} \mathrm{MgCl}_{2}$, buffered with $0.5 \times \mathrm{TBE}$, to the same cuvette while recording the emission spectra.

Single-Molecule Fluorescence Measurements. Single-molecule FRET measurements of individual diffusing DNA origamis were performed in LabTek chamber slides (Thermo Scientific) that were cleaned with $1 \mathrm{M} \mathrm{KOH}$ and passivated with BSA $(10 \mathrm{mg} / \mathrm{mL})$. DNA origami structures were diluted in a buffer consisting of $1 \times \mathrm{PBS}, 12.5 \mathrm{mM} \mathrm{MgCl}_{2}$ for stabilization of the origami and $2 \mathrm{mM}$ trolox/troloxquinone, $1 \%(\mathrm{w} / \mathrm{w})$ glucose, and $10 \%(\mathrm{v} / \mathrm{v})$ of GOC $(1 \mathrm{mg} / \mathrm{mL}$ glucose oxidase, $0.4 \%(\mathrm{v} / \mathrm{v})$ catalase $(50 \mu \mathrm{g} / \mathrm{mL}), 30 \%$ glycerol, and $12.5 \mathrm{mM}$ $\mathrm{KCl}$ in $50 \mathrm{mM}$ TRIS $\mathrm{pH} 7.5)^{27}$ for stabilization of the fluorophores. Burst measurements were carried out on a custom-built confocal fluorescence microscopy setup. A diode laser with $80 \mathrm{MHz}$ pulse frequency (640 nm, LDH-D-C-640, Picoquant) and a $532 \mathrm{~nm} \mathrm{cw}$ laser (Sapphire LP $532 \mathrm{~nm} 100$ $\mathrm{mW}$, Coherent) were alternated by an acousto-optical tunable filter (AOTFnc-VIS, AA optoelectronic) with $100 \mu$ s period. The laser beams were coupled into an oil-immersion objective (UPlanSApo 60XO/1.35 NA, Olympus) that is incorporated in an inverted microscope body (Olympus IX-71). The emission light was collected by the same objective and was separated from the excitation light by a dual-band dichroic beam splitter (z532/633 AHF) and focused onto a $50 \mu \mathrm{m}$ pinhole (Linos). The emission of $\mathrm{Cy} 3$ and $\mathrm{Cy} 5$ was split spectrally by a singleband dichroic mirror (640 DCXR, AHF) and focused onto two avalanche photo diodes ( $\tau$-SPAD-100, Picoquant) with appropriate filtering (Cy3 emission, Brightline HC582/75, AHF and RazorEdge LP 532, Semrock; Cy5 emission, Bandpass ET 700/75m, AHF and RazorEdge LP 647, Semrock). The detector signals were registered with a single photon counting PC card (SPC-830, Becker \& Hickl) and evaluated using custom-made LabVIEW (National Instruments) software (see SI Methods for details on data analysis).

Model. We modeled the energy transfer from the antenna complex to the common acceptor core using a set of rate equations governing the dynamics of the populations of the donor and acceptor chromophores, under external laser excitation and hetero-FRET interaction. This treatment assumes that only one particle, one excitonic quasiparticle in this case, is present in the system at any time, which is valid for the low excitation conditions under which the experiments have been carried out.

For a single donor-acceptor pair, the antenna effect $\left(A E_{A}^{D}\right)$ is given by (see SI Note S8 for the full derivation)

$$
\mathrm{AE}_{\mathrm{A}}^{\mathrm{D}}=\frac{\Phi_{\mathrm{D}}}{\Phi_{\mathrm{A}}} E(R)
$$

where $\Phi_{\mathrm{D}}\left(\Phi_{\mathrm{A}}\right)$ is proportional to the molar extinction coefficient of the donor (acceptor) dye $\Xi_{\mathrm{D}}\left(\lambda_{\mathrm{D}}\right) \quad\left[\Xi_{\mathrm{D}}\left(\lambda_{\mathrm{A}}\right)\right]$ dependent on the excitation wavelength $\lambda_{\mathrm{D}}\left(\lambda_{\mathrm{A}}\right)$; see also SI Note $S 8$, and $E(R)$ the FRET efficiency

$$
E(R)=R_{0}^{6} /\left(R_{0}^{6}+R^{6}\right)
$$

with $R_{0}$ the Förster radius, that is, the donor-acceptor separation corresponding to a FRET efficiency $E(R)$ equal to $50 \%$.

When more than one donor is present, as in the ring antenna system examined here, the cumulative antenna effect $\left(\mathrm{AE}_{\text {tot }}\right)$ simply scales with the total number $N$ of donor dyes $D_{i}(i=1$, ..., $N)$, if these are identical and located at a same distance from the acceptor $A$

$$
\mathrm{AE}_{\text {tot }}=N \mathrm{AE}_{\mathrm{A}}^{\mathrm{D}}
$$

In this case, we have not considered the homo-FRET interaction between identical donors. It can be demonstrated that the energy transfer between identical dyes located at the same distance from the common acceptor does not alter the net antenna effect (SI Note S8).

For two donors $D_{1}$ and $D_{2}$ nearly equally spaced from the common acceptor $A$ with distances $R_{1}$ and $R_{2}$, respectively, we can introduce an average donor-acceptor separation $\bar{R}$ so that $R_{1}=\bar{R}+\delta R \gtrsim R_{2}=\bar{R}-\delta R$. In this case, the cumulative antenna effect, $\mathrm{AE}_{\mathrm{A}}^{D_{1}}+\mathrm{AE}_{\mathrm{A}}^{D_{2}}$ can be expressed in terms of $\bar{R}$ (see also SI Note S10)

$$
\begin{aligned}
\mathrm{AE}_{\text {tot }}(\bar{R}) & =\frac{\alpha}{1+\beta(\bar{R}+\delta R)^{6}}+\frac{\alpha}{1+\beta(\bar{R}-\delta R)^{6}} \\
& \approx \frac{2 \alpha}{1+\beta \bar{R}^{6}}+O\left(\delta R^{2}\right)
\end{aligned}
$$

where $\alpha=\Phi_{\mathrm{D}} / \Phi_{\mathrm{A}}$ and $\beta=1 / R_{0}^{6}$.

We have used the experimental efficiencies measured by single-molecule spectroscopy (SI Figure S3), to evaluate the two distinct donor-acceptor distances (D1-A and $D 2-A$ ). Using eq 3 with a Förster radius $R_{0}=5.4 \mathrm{~nm},{ }^{17}$ we get $R_{D_{1} A}=$ $5.2 \mathrm{~nm}$ and $R_{D_{2} A}=5.6 \mathrm{~nm}$.

The experimental values of the antenna effect as a function of the number of donors (Figure 2d) have been fitted using eq 4 . In this case, we have assumed an average donor-acceptor efficiency $E(\bar{R}), \bar{R}=\left(R_{D_{1} A}+R_{D_{1} A}\right) / 2$ (see SI Note S10) and fitted the experimental antenna effects $(y)$ with the linear expression $y=\tilde{\alpha} x+q, x=N E(\bar{R})(N=1, \ldots, 6)$ and $\tilde{\alpha}=\Phi_{\mathrm{D}} /$ $\Phi_{\mathrm{A}}$. From the linear fit, we get $\tilde{\alpha}=\Phi_{\mathrm{D}} / \Phi_{\mathrm{A}}=0.606$ and $q=$ 0.006 . Then, with $\tilde{\alpha}$ obtained from the linear fit and using $R_{0}=$ $5.4 \mathrm{~nm}$, we compare the theoretical antenna effect (eq 5) evaluated as a function of the donor-acceptor separation (in the two-donor configuration) with the experimental values (see Figure 2e).

\section{ASSOCIATED CONTENT}

\section{S Supporting Information}

The Supporting Information is available free of charge on the ACS Publications website at DOI: 10.1021/acs.nanolett.5b05139.

DNA origami design, fluorophore arrangement and attachment, spectroscopic properties of $\mathrm{Cy} 3$ and $\mathrm{Cy} 5$, 
energy transfer efficiency of single donor-acceptor pairs, calculation of antenna effect in ensemble fluorescence measurements, magnesium dependent fluorescence quenching, histograms from single-molecule fluorescence measurements, comparison of antenna effect and energy transfer efficiency between single-molecule and ensemble measurements, detailed derivation of model and further theoretical considerations, and supplementary methods. (PDF)

\section{AUTHOR INFORMATION}

\section{Corresponding Authors}

*E-mail: ufk20@cam.ac.uk.

*E-mail: ac307@cam.ac.uk.

\section{Author Contributions}

M.A.P. and S.E. initiated the project. U.F.K. and A.W.C. conceived the work. U.F.K. and E.A.H. designed the experiments. E.A.H. and P.M. prepared the DNA origami samples, performed the ensemble spectroscopic measurements, and analyzed the data. B.W. and L.H. performed the single-molecule spectroscopic measurements on the DNA origami samples and analyzed the related data. P.T. supervised the single-molecule measurements. A.W.C. and C.C. performed the simulations based on Förster theory used to model the experimental data. E.A.H. and C.C. wrote the manuscript with inputs from all authors.

\section{Notes}

The authors declare no competing financial interest.

\section{ACKNOWLEDGMENTS}

A.W.C. acknowledges support from the Winton Programme for the Physics of Sustainability. U.F.K. was partly supported by an ERC starting grant (PassMembrane, EU 261101). E.A.H. acknowledges support from Janggen-Pöhn Stiftung and the Schweizerischer Nationalfonds (SNF). P.T. acknowledges support by a starting grant (SiMBA, EU 261162) of the European Research Council (ERC). B.W. gratefully acknowledges support by the Braunschweig International Graduate School of Metrology B-IGSM and the DFG Research Training Group GrK1952/1 "Metrology for Complex Nanosystems". P.M. thankfully acknowledges the support of the EPSRC Centre for Doctoral Training in Sensor Technologies and Applications EP/L015889/1.

\section{REFERENCES}

(1) Blankenship, R. E. Molecular Mechanisms of Photosynthesis; Blackwell Publishing Ltd.: Oxford, U. K., 2008.

(2) Scholes, G. D.; Fleming, G. R; Olaya-Castro, A.; van Grondelle, R. Nat. Chem. 2011, 3, 763-774.

(3) Heller, M. J.; Tullis, R. H. Nanotechnology 1991, 2, 165.

(4) Stein, I. H.; Schüller, V.; Böhm, P.; Tinnefeld, P.; Liedl, T. ChemPhysChem 2011, 12, 689-695.

(5) Stein, I. H.; Steinhauer, C.; Tinnefeld, P. J. Am. Chem. Soc. 2011, 133, 4193-4195.

(6) Hannestad, J. K.; Sandin, P.; Albinsson, B. J. Am. Chem. Soc. 2008, 130, 15889-15895.

(7) Buckhout-White, S.; Spillmann, C. M.; Algar, W. R.; Khachatrian, A.; Melinger, J. S.; Goldman, E. R.; Ancona, M. G.; Medintz, I. L. Nat. Commun. 2014, 5, 5615.

(8) Dutta, P. K.; Varghese, R.; Nangreave, J.; Lin, S.; Yan, H.; Liu, Y. J. Am. Chem. Soc. 2011, 133, 11985-11993.

(9) Albinsson, B.; Hannestad, J. K.; Börjesson, K. Coord. Chem. Rev. 2012, 256, 2399-2413.
(10) Woller, J. G.; Hannestad, J. K.; Albinsson, B. J. Am. Chem. Soc 2013, 135, 2759-2768.

(11) Dutta, P. K.; Levenberg, S.; Loskutov, A.; Jun, D.; Saer, R.; Beatty, J. T.; Lin, S.; Liu, Y.; Woodbury, N. W.; Yan, H. J. Am. Chem. Soc. 2014, 136, 16618-16625.

(12) Pan, K.; Boulais, E.; Yang, L.; Bathe, M. Nucleic Acids Res. 2014, 42, 2159-2170.

(13) Rothemund, P. W. K. Nature 2006, 440, 297-302.

(14) Castro, C. E.; Kilchherr, F.; Kim, D.-N.; Shiao, E. L.; Wauer, T.; Wortmann, P.; Bathe, M.; Dietz, H. Nat. Methods 2011, 8, 221-229.

(15) Liedl, T.; Högberg, B.; Tytell, J.; Ingber, D. E.; Shih, W. M. Nat. Nanotechnol. 2010, 5, 520-524.

(16) Hu, X.; Damjanović, A.; Ritz, T.; Schulten, K. Proc. Natl. Acad. Sci. U. S. A. 1998, 95, 5935-5941.

(17) Lee, S.; Lee, J.; Hohng, S. PLoS One 2010, 5, e12270.

(18) Khan, Y. R.; Dykstra, T. E.; Scholes, G. D. Chem. Phys. Lett. 2008, 461, 305-309.

(19) Olaya-Castro, A.; Scholes, G. D. Int. Rev. Phys. Chem. 2011, 30, 49-77.

(20) Li, C.-Y.; Hemmig, E. A.; Kong, J.; Yoo, J.; Hernández-Ainsa, S.; Keyser, U. F.; Aksimentiev, A. ACS Nano 2015, 9, 1420-1433.

(21) Brousmiche, D. W.; Serin, J. M.; Fréchet, J. M. J.; He, G. S.; Lin, T.-C.; Chung, S.-J.; Prasad, P. N.; Kannan, R.; Tan, L.-S. J. Phys. Chem. B 2004, 108, 8592-8600.

(22) Creatore, C.; Parker, M. A.; Emmott, S.; Chin, A. W. Phys. Rev. Lett. 2013, 111, 253601.

(23) Higgins, K. D. B.; Benjamin, S. C.; Stace, T. M.; Milburn, G. J.; Lovett, B. W.; Gauger, E. M. Nat. Commun. 2014, 5, 4705.

(24) Zhang, Y.; Oh, S.; Alharbi, F. H.; Engel, G. S.; Kais, S. Phys. Chem. Chem. Phys. 2015, 17, 5743-5750.

(25) Creatore, C.; Chin, A. W.; Parker, M. A.; Emmott, S. Front. Mater. 2015, 2, 6.

(26) Asanuma, H.; Fujii, T.; Kato, T.; Kashida, H. J. Photochem. Photobiol., C 2012, 13, 124-135.

(27) Cordes, T.; Vogelsang, J.; Tinnefeld, P. J. Am. Chem. Soc. 2009, 131, 5018-5019. 